\title{
Lorentz Invariant Berry Phase for a Perturbed Relativistic Four Dimensional Harmonic Oscillator
}

\author{
Y. Bachar, ${ }^{1}$ R. I. Arshansky, ${ }^{2}$ L. P. Horwitz,${ }^{3,1,4}$ and I. Aharonovich ${ }^{1}$ \\ ${ }^{1}$ Dept. of Physics, Bar Ilan Univeristy* \\ ${ }^{2}$ Givat Zorfatit, Etzel 12/14, Jerusalem 9754 \\ ${ }^{3}$ School of Physics and Astronomy, Tel-Aviv University \\ ${ }^{4}$ Dept. of Physics, Ariel University in the Shomron
}

\begin{abstract}
We show the existence of Lorentz invariant Berry phases generated, in the Stueckleberg-HorwitzPiron manifestly covariant quantum theory (SHP), by a perturbed four dimensional harmonic oscillator. These phases are associated with a fractional perturbation of the azimuthal symmetry of the oscillator. They are computed numerically by using time independent perturbation theory and the definition of the Berry phase generalized to the framework of SHP relativistic quantum theory.

PACS numbers: 03.65.Ta, 03.65.Vf, 03.65.Pm, 03.30.+p
\end{abstract}

Keywords: Relativistic Quantum Mechanics; Geometric Phase

* yossi_dk@yahoo.com 
A manifestly covariant quantum mechanics was formulated by E. C. G. Stueckelberg [8] in 1941. He studied this theory for the case of a single particle in an external field. He considered the phenomenon of pair annihilation and creation as a manifestation of the development, in each case, of a single world line that curves in such a way that one part runs forward and one part runs backward in time, and above the turning point the line does not pass at all. This configuration was considered by Stueckelberg to represent pair annihilation. To describe such a curve, parametrization by the variable $t$ is ineffective, since the trajectory is not single valued. He therefore introduced a parametric description, with parameter $\tau$, along the world line. Hence one branch of the curve is generated by motion in the positive sense of $t$ as a function of increasing $\tau$, and the other branch by motion in the negative sense of $t$, i.e., the antiparticle.

The motion, in space-time, of the point generating the world line, which we shall call an event (which has the properties of space-time position and energy momentum), is governed in the classical case by the Hamilton equations in space-time

$$
\frac{\mathrm{d} x^{\mu}}{\mathrm{d} \tau}=\frac{\partial K}{\partial p_{\mu}}, \quad \frac{\mathrm{d} p^{\mu}}{\mathrm{d} \tau}=-\frac{\partial K}{\partial x_{\mu}}
$$

where $x^{\mu}=(t, \vec{x}), p^{\mu}=(E, \vec{p})\left[\right.$ we take $c=1$ and $\left.g_{\mu \nu}=(-1,1,1,1)\right]$ and the evolution generator $K$ is a function of the canonical variables $x_{\mu}, p_{\mu}$ which satisfies the Poission brackets $\left\{x_{\mu}, p_{\nu}\right\}=g_{\mu \nu}$. For the special case of free motion,

$$
K_{0}=\frac{p^{\mu} p_{\mu}}{2 M}
$$

where $M$ is an intrinsic parameter assigned to the generic event, and hence

$$
\frac{\mathrm{d} x^{\mu}}{\mathrm{d} \tau}=\frac{p^{\mu}}{M}
$$

It then follows that

$$
\frac{\mathrm{d} \vec{x}}{\mathrm{~d} t}=\frac{\vec{p}}{E}
$$

consistent with standard relativistic kinematics. We note, however, that the mass squared $m^{2}=-p^{\mu} p_{\mu}$ is a dynamical variable since $\vec{p}$ and $\mathrm{E}$ are considered to be kinematically independent, and therefore it is not taken to be equal to an a priori given constant. The set 
of values taken by $m^{2}$ in a particular dynamical context is determined by initial conditions and the dynamical equations.

In the quantum theory, $\vec{x}, t$ (and $\vec{p}, E$ ) denote operators satisfying the commutation relations (we take $\hbar=1$ )

$$
\left[x^{\mu}, p^{\nu}\right]=i g^{\mu \nu}
$$

The state of a one-event system is described by a wave function $\psi_{\tau}(x) \in L^{2}\left(R^{4}\right)$, a complex Hilbert space with measure $\mathrm{d}^{4} x=\mathrm{d}^{3} x \mathrm{~d} t$ satisfying the equation

$$
i \frac{\partial \psi_{\tau}(x)}{\partial \tau}=K \psi_{\tau}(x)
$$

This equation, designed to provide a manifestly covariant description of relativistic phenomena, is similar in form to the non-relativistic Schrödinger equation. Although free motion is determined by the operator form of $K_{0}$ of Eq. (2), i.e., the d'Alembertian, which is hyperbolic $\left(p_{\mu} p^{\mu} \equiv-\partial_{\mu} \partial^{\mu}\right.$ instead of the elliptic operator $\left.\vec{p}^{2} \equiv-\nabla^{2}\right)$, the same methods may be used for studying Eq. (6) as for the non-relativistic Schrodinger equation.

In 1973 Horwitz and Piron [6] generalized the Stueckelberg theory by assuming that for the treatment of systems of more than one event (generating world lines of more than one particle), there is a single universal $\tau$ which parametrizes the motion of all of the particles of the many body system (we denote this generalized theory by SHP). They assumed the unperturbed evolution generator to be of the form

$$
K_{0}=\sum_{i=1}^{N} \frac{p_{i}^{\mu} p_{i \mu}}{2 M_{i}}
$$

There is a class of model systems, for which solutions can be achieved using straightforward methods, which involve only effective action-at-a-distance (direct action) potentials, where the evolution generator is of the form

$$
K=\sum_{i=1}^{N} \frac{p_{i}^{2}}{2 M_{i}}+V\left(x_{1}, x_{2}, \ldots, x_{N}\right)
$$

Note that in this case the potential function enters into the dynamical evolution equation as a term added to the generator of the free motion, and therefore corresponds to a spacetime coordinate-dependent interaction mass.

In 1989 Horwitz and Arshansky [1] demonstrated the existence of bound state solutions for the quantum case by solving the dynamical equation (6) associated with the dynamical 
evolution operator (8). The two-body potential function, which they chose was of the form $V(\rho)=\frac{k}{\rho}$, where

$$
\rho=\sqrt{\left(x_{1}^{\mu}-x_{2}^{\mu}\right)\left(x_{1 \mu}-x_{2 \mu}\right)} \equiv \sqrt{\left(x_{1}-x_{2}\right)^{2}}
$$

and $x_{1}^{\mu}-x_{2}^{\mu}$ is spacelike, and $k$ is a coupling constant. For the two body case, the Hamiltonian can be written as a sum of center of mass and relative (reduced motion part) using exactly the same procedure as the nonrelativistic case (the Hamiltonian has the same quadratic form), with potential $V(x)$, where $x=x_{1}-x_{2}[1]$.

Cook solved this problem [4] with support for $x^{\mu}$ in the full spacelike region and obtained a spectrum which disagreed with the nonrelativistic Schrodinger spectrum. Zmuidzinas [9] showed, however that there is no complete orthogonal set in the full spacelike region, but there are complete sets in the two manifolds (defined as in Figure 1), by separating the spacelike region into two parts for which $x^{2}+y^{2}-t^{2}>0$ (The RMS) and $x^{2}+y^{2}-t^{2}<0$. Then Arshansky and Horwitz defined wavefunctions with support in RMS to obtain a spectrum which agrees with the nonrelativistic Schrodinger solutions.

To describe the RMS we use the definition

$$
x^{0}=\rho \sin \theta \sinh \beta \quad x^{1}=\rho \sin \theta \cos \phi \cosh \beta \quad x^{2}=\rho \sin \theta \sin \phi \sinh \beta \quad x^{3}=\rho \cos \theta
$$

These variables span the entire RMS. The normalizable solutions of the StueckelbergSchrodinger equation vanish identically on the boundaries, so there is no tunneling to the second spacelike region (II) or the light cone.

When they applied the method of treating the relativistic quantum two body problem to the case of the four dimensional harmonic oscillator, they took the reduced Hamiltonian to be

$$
\begin{aligned}
K=\frac{p_{\mu} p^{\mu}}{2 m}+\frac{1}{2} k x_{\mu} x^{\mu}=- & \frac{1}{2 m} \partial_{\mu} \partial^{\mu}+\frac{1}{2} m w^{2} \rho^{2} \\
& =\frac{1}{2 m}\left[-\frac{\partial^{2}}{\partial \rho^{2}}-\frac{3}{\rho} \frac{\partial}{\partial \rho}+\frac{\Lambda}{\rho^{2}}\right]+\frac{1}{2} m w^{2} \rho^{2}
\end{aligned}
$$

They obtained the complete set of eigenvectors, represented by the $\tau$ independent wave functions 


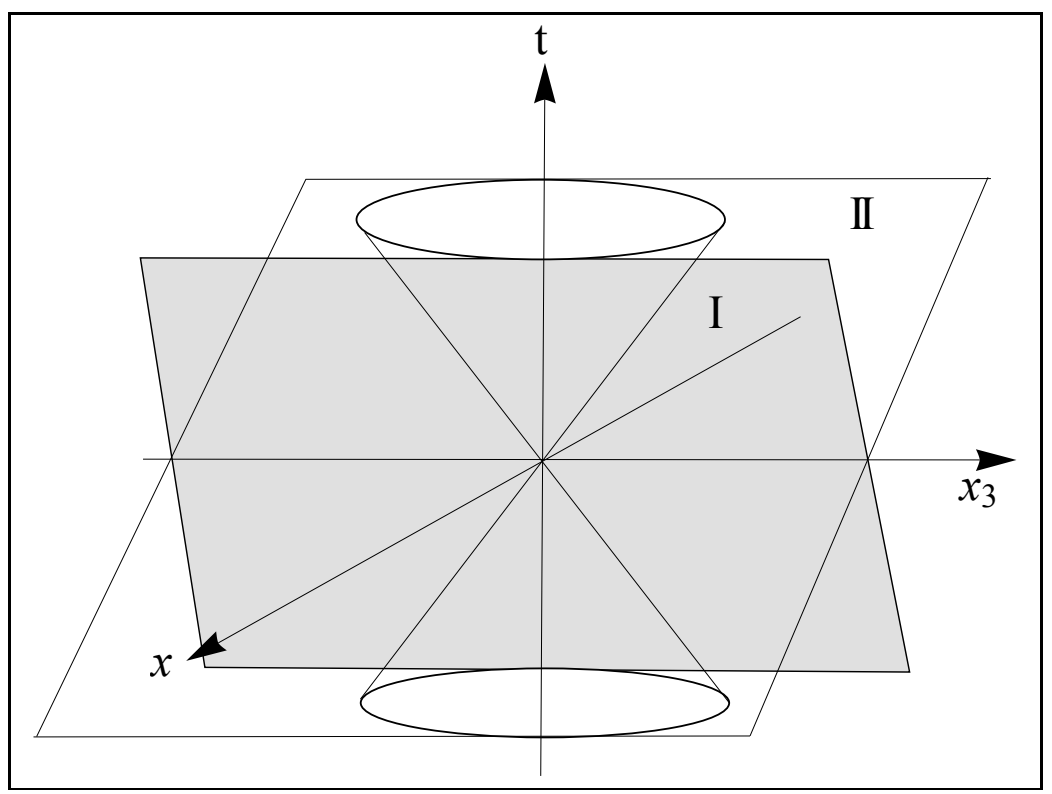

FIG. 1. RMS-Reduced Minkowski Space (I) consists of the space-time points external (in spacelike direction) to the two hyperplanes tangent to the light cone that is oriented along the $\mathrm{z}$ axis (the direction must be chosen to define this space). The second subregion (II) consists of the space-time points in the sector interior (timelike direction) to these hyperplanes, but excluding the light cone. In this figure the configuration is shown schematically by folding the two space axes $\mathrm{x}, \mathrm{y}$ together (defining the coordinate $x_{\perp}$ ). Both subregions are invariant under an $O(2,1)$ subgroup of $O(3,1)$

$$
\begin{aligned}
\psi_{n_{a} l n m}(\phi, \beta, \theta, \rho)=\frac{1}{2 \pi} & e^{i(m+1 / 2) \phi} \times \sqrt{n} \sqrt{\Gamma(1+m+n) / \Gamma(1+m-n)} \times \\
& \times\left(1-\tanh ^{2} \beta\right)^{\frac{1}{4}} P_{m}^{-n}(\tanh \beta) \times\left(1-\cos ^{2} \theta\right)^{-\frac{1}{4}} \times \\
& \times P_{l}^{n}(\cos \theta) \frac{1}{\sqrt{\rho}}\left(\frac{m w \rho^{2}}{\hbar}\right)^{l / 2} e^{-\frac{m w \rho^{2}}{2 \hbar}} L_{n_{a}}^{l+1 / 2}\left(\frac{m w \rho^{2}}{\hbar}\right)
\end{aligned}
$$

We now study the effect of an adiabatic perturbation on the Hamiltonian (11) on the wavefunction (12).

The idea of the geometric phase proposed by Berry [3], [5] in 1984 asserts that under adiabatic processes the wave function of a system picks up a phase factor that can be found in the nonrelativistic case by the integral

$$
\gamma_{n}(t) \equiv i \int_{0}^{t}\left\langle\psi_{n}\left(t^{\prime}\right) \mid \frac{\partial \psi_{n}\left(t^{\prime}\right)}{\partial t}\right\rangle d t^{\prime}
$$


where $\psi_{n}$ is the nth eigenvector of the slowly changing Hamiltonian (for which one can prove the adiabatic theorem), $t$ is the time between the initial and final values of the time dependent parameters of the Hamiltonian and $\frac{\partial \psi_{n}}{\partial t}$ corresponds to the change in the wavefunction under time variation of the parameters. To construct a manifestly covariant form of (13), we have to replace the wavefunction by solutions of Eq.(6), and the variable $t$ by the variable $\tau$ which is the evolution time according to SHP quantum mechanics. Suppose there are N $\tau$ dependent parameters: $R_{1}(\tau), R_{2}(\tau), \ldots . . R_{N}(\tau)$ in the Hamiltonian of a given problem; then

$$
\frac{\partial \psi_{n}}{\partial \tau}=\frac{\partial \psi_{n}}{\partial R_{1}} \frac{d R_{1}}{d \tau}+\frac{\partial \psi_{n}}{\partial R_{2}} \frac{d R_{2}}{d \tau}+\ldots \ldots+\frac{\partial \psi_{n}}{\partial R_{N}} \frac{d R_{N}}{d \tau}=\left(\nabla_{\vec{R}} \psi_{n}\right) \cdot \frac{d \vec{R}}{d \tau}
$$

where

$$
\vec{R}=\left(R_{1}, R_{2}, \ldots, R_{N}\right)
$$

We then have

$$
\gamma_{n}(\tau)=i \int_{\overrightarrow{R_{i}}}^{\overrightarrow{R_{f}}}\left\langle\psi_{n} \mid \nabla_{\vec{R}} \psi_{n}\right\rangle \cdot d \vec{R}
$$

Now, if the Hamiltonian returns to its original form after a time $\tau=T$, then the geometric phase is

$$
\gamma_{n}(T)=i \oint\left\langle\psi_{n} \mid \nabla_{\vec{R}} \psi_{n}\right\rangle \cdot d \vec{R}
$$

We wish to demonstrate the realization of an invariant Berry phase in the example of the four dimensional oscillator. For that purpose we add a perturbation to the Hamiltonian (11) which breaks the hyperangular symmetry of the Hamiltonian. Since the complex valued matrix elements necessary to develop a dynamical phase arise in this example from the $\phi$ dependance, one must perturb the azimuthal symmetry with a fractional coefficient, as we shall see below. As a simple example we take the perturbed Hamiltonian to be

$$
\begin{aligned}
K=\frac{1}{2 m} & {\left[-\frac{\partial^{2}}{\partial \rho^{2}}-\frac{3}{\rho} \frac{\partial}{\partial \rho}+\frac{\Lambda}{\rho^{2}}\right]+\frac{1}{2} m w^{2} \rho^{2}+} \\
& +\varepsilon_{1} \rho^{2} \sin ^{2} \theta \cos ^{2}((2 / 3) \phi) \cosh ^{2} \beta+ \\
& +\varepsilon_{2} \rho^{2} \sin ^{2} \theta \sin ^{2}((2 / 3) \phi) \cosh ^{2} \beta+ \\
& +\varepsilon_{3} \rho^{2} \cos ^{2} \theta-\varepsilon_{0} \rho^{2} \sin ^{2} \theta \sinh ^{2} \beta
\end{aligned}
$$

where $\varepsilon_{0}, \varepsilon_{1}, \varepsilon_{2}, \varepsilon_{3}$ are the small parameters of the perturbation.

Now using degenerate time independent perturbation theory, we calculate the first order 
correction to the wave function (12).

The new wave function produced by the Hamiltonian (18) is equal to a linear combination of the wave function (12) and its first order correction, which is given by

$$
\psi_{n_{a}^{\prime} l^{\prime} n^{\prime} m^{\prime}}^{(1)}=\sum_{n_{a}, l \neq n_{a}^{\prime}, l^{\prime}} \frac{\left\langle\psi_{n_{a} l n m}|V| \psi_{n_{a}^{\prime} l^{\prime} n^{\prime} m^{\prime}}\right\rangle}{K_{a}^{\prime}-K_{a}} \psi_{n_{a} l n m}
$$

where $\mathrm{V}$ is the perturbation given in (18), and $K_{a}$ is the eigenvalue of the unperturbed Hamiltonian (11),

$$
K_{a}=\hbar \omega\left(l+2 n_{a}+\frac{3}{2}\right)
$$

Now suppose $\varepsilon_{0}$ and $\varepsilon_{3}$ are equal to zero so that Eq.(19) becomes

$$
\begin{aligned}
\psi_{n_{a}^{\prime} l^{\prime} n^{\prime} m^{\prime}}^{(1)}= & \varepsilon_{1} \sum_{n_{a}, l \neq n_{a}^{\prime}, l^{\prime}} \frac{\left\langle\psi_{n_{a} l n m}\left|\rho^{2} \sin ^{2} \theta \cos ^{2}((2 / 3) \phi) \cosh ^{2} \beta\right| \psi_{n_{a}^{\prime} l^{\prime} n^{\prime} m^{\prime}}\right\rangle}{\left[l^{\prime}-l+2\left(n_{a}^{\prime}-n_{a}\right)\right] \hbar \omega} \psi_{n_{a} l n m} \\
& +\varepsilon_{2} \sum_{n_{a}, l \neq n_{a}^{\prime}, l^{\prime}} \frac{\left\langle\psi_{n_{a} l n m}\left|\rho^{2} \sin ^{2} \theta \sin ^{2}((2 / 3) \phi) \cosh ^{2} \beta\right| \psi_{n_{a}^{\prime} l^{\prime} n^{\prime} m^{\prime}}\right\rangle}{\left[l^{\prime}-l+2\left(n_{a}^{\prime}-n_{a}\right)\right] \hbar \omega} \psi_{n_{a} l n m}
\end{aligned}
$$

or

$$
\psi_{n_{a}^{\prime} l^{\prime} n^{\prime} m^{\prime}}^{(1)}=\varepsilon_{1} \psi_{n_{a}^{\prime} l^{\prime} n^{\prime} m^{\prime}}^{\prime}+\varepsilon_{2} \psi_{n_{a}^{\prime} l^{\prime} n^{\prime} m^{\prime}}^{\prime \prime}
$$

The simplest way to demonstrate the existence of a geometric phase is by letting each one of the indices $\left(n_{a}, n_{a}^{\prime}, l, l^{\prime}, n, n^{\prime}, m, m^{\prime}\right)$ take the value 2 or 3 . In that way our perturbation can be represented as a $16 \times 16$ matrix. The Hilbert space of the problem is a 16 dimensional space composed of four 4 dimensional subspaces, each subspace corresponding to one of the four energy eigenvalues. The next table summarizes all the possible permutations (note that some of the entries in this table, those for which $n>l$, vanish identically and therefore do not appear in any calculation. We list them for completeness among the indices.) 


\begin{tabular}{|c|c|c|c|c|}
\hline$n_{a}$ & \begin{tabular}{|l|l|}
$l$ & $n$ \\
\end{tabular} & $m$ & & $K_{a i}(\hbar \omega)$ \\
\hline 2 & 22 & & 1 & 7.5 \\
\hline 2 & $\begin{array}{lll}2 & 2\end{array}$ & 3 & 2 & \\
\hline 2 & \begin{tabular}{|l|l|}
2 & 3 \\
\end{tabular} & 2 & 3 & \\
\hline 2 & \begin{tabular}{|l|l|}
2 & 3
\end{tabular} & 3 & 4 & \\
\hline 2 & $\begin{array}{lll}3 & 2 \\
\end{array}$ & 2 & 5 & 8.5 \\
\hline 2 & \begin{tabular}{|l|l|}
3 & 2 \\
\end{tabular} & 3 & 6 & \\
\hline 2 & \begin{tabular}{|l|l|}
3 & 3 \\
\end{tabular} & 2 & 7 & \\
\hline 2 & \begin{tabular}{|l|l|}
3 & 3 \\
\end{tabular} & 3 & 8 & \\
\hline 3 & 22 & 2 & 9 & 9.5 \\
\hline 3 & $\begin{array}{lll}2 & 2 \\
\end{array}$ & 3 & 10 & \\
\hline 3 & 23 & 2 & 11 & \\
\hline 3 & 23 & & 12 & \\
\hline 3 & \begin{tabular}{|l|l|}
3 & 2 \\
\end{tabular} & 2 & 13 & 10.5 \\
\hline 3 & \begin{tabular}{l|l|l|}
3 & 2 \\
\end{tabular} & 3 & 14 & \\
\hline 3 & $\begin{array}{lll}3 & 3\end{array}$ & & 15 & \\
\hline 3 & 33 & & 16 & \\
\hline
\end{tabular}

Let $D_{1}$ be the subspace of all the eigenfunctions that correspond to the eigenvalue $K_{a 1}$, so we can write

$D_{1}=\operatorname{span}\left\{\psi_{1}, \psi_{2}, \psi_{3}, \psi_{4}\right\}$

and similarly

$D_{2}=\operatorname{span}\left\{\psi_{5}, \psi_{6}, \psi_{7}, \psi_{8}\right\}$

$D_{3}=\operatorname{span}\left\{\psi_{9}, \psi_{10}, \psi_{11}, \psi_{12}\right\}$

$D_{4}=\operatorname{span}\left\{\psi_{13}, \psi_{14}, \psi_{15}, \psi_{16}\right\}$

For example, let us find the first order correction of $\psi_{1} \in D_{1}$; we chose $\psi_{1}$ because this is one of the nonzero ground states (we will see later why it is nonzero) of the oscillator (along with $\psi_{2}$ ).

Substituting the matrix elements of V' and V" which we shall compute numerically later, 
where

$$
V^{\prime}=\rho^{2} \sin ^{2} \theta \cos ^{2}((2 / 3) \phi) \cosh ^{2} \beta
$$

and

$$
V^{\prime \prime}=\rho^{2} \sin ^{2} \theta \sin ^{2}((2 / 3) \phi) \cosh ^{2} \beta
$$

while $\hbar=6.626 \cdot 10^{-34} \mathrm{~J} \cdot \mathrm{s}, \mathrm{m}=9.109 \cdot 10^{-31} \mathrm{~kg}$ (electron mass), and $\omega=240.4 \mathrm{MHz}$.

the value of $\omega$ is determined by the wish to obtain an order one value for the Berry phase (up to the scale of the perturbation squared).

According to Eq. (21),

$$
\begin{aligned}
& \psi_{1}^{(1)}=\varepsilon_{1}[ \frac{\left\langle\psi_{5}\left|V^{\prime}\right| \psi_{1}\right\rangle}{K_{a 1}-K_{a 5}} \psi_{5}+\frac{\left\langle\psi_{6}\left|V^{\prime}\right| \psi_{1}\right\rangle}{K_{a 1}-K_{a 6}} \psi_{6}+\frac{\left\langle\psi_{7}\left|V^{\prime}\right| \psi_{1}\right\rangle}{K_{a 1}-K_{a 7}} \psi_{7} \\
&+\frac{\left\langle\psi_{8}\left|V^{\prime}\right| \psi_{1}\right\rangle}{K_{a 1}-K_{a 8}} \psi_{8}+\frac{\left\langle\psi_{9}\left|V^{\prime}\right| \psi_{1}\right\rangle}{K_{a 1}-K_{a 9}} \psi_{9}+\frac{\left\langle\psi_{10}\left|V^{\prime}\right| \psi_{1}\right\rangle}{K_{a 1}-K_{a 10}} \psi_{10} \\
&+\frac{\left\langle\psi_{11}\left|V^{\prime}\right| \psi_{1}\right\rangle}{K_{a 1}-K_{a 11}} \psi_{11}+\frac{\left\langle\psi_{12}\left|V^{\prime}\right| \psi_{1}\right\rangle}{K_{a 1}-K_{a 12}} \psi_{12}+\frac{\left\langle\psi_{13}\left|V^{\prime}\right| \psi_{1}\right\rangle}{K_{a 1}-K_{a 13}} \psi_{13} \\
&\left.+\frac{\left\langle\psi_{14}\left|V^{\prime}\right| \psi_{1}\right\rangle}{K_{a 1}-K_{a 14}} \psi_{14}+\frac{\left\langle\psi_{15}\left|V^{\prime}\right| \psi_{1}\right\rangle}{K_{a 1}-K_{a 15}} \psi_{15}+\frac{\left\langle\psi_{16}\left|V^{\prime}\right| \psi_{1}\right\rangle}{K_{a 1}-K_{a 16}} \psi_{16}\right] \\
&+\varepsilon_{2}\left[\frac{\left\langle\psi_{5}\left|V^{\prime \prime}\right| \psi_{1}\right\rangle}{K_{a 1}-K_{a 5}} \psi_{5}+\frac{\left\langle\psi_{6}\left|V^{\prime \prime}\right| \psi_{1}\right\rangle}{K_{a 1}-K_{a 6}} \psi_{6}+\frac{\left\langle\psi_{7}\left|V^{\prime \prime}\right| \psi_{1}\right\rangle}{K_{a 1}-K_{a 7}} \psi_{7}\right. \\
&\left.+\frac{\left\langle\psi_{14}\left|V^{\prime \prime}\right| \psi_{1}\right\rangle}{K_{a 1}-K_{a 14}} \psi_{14}+\frac{\left\langle\psi_{15}\left|V^{\prime \prime}\right| \psi_{1}\right\rangle}{K_{a 1}-K_{a 15}} \psi_{15}+\frac{\left\langle\psi_{16}\left|V^{\prime \prime}\right| \psi_{1}\right\rangle}{K_{a 1}-K_{a 16}} \psi_{16}\right] \\
&+\frac{\left\langle\psi_{8}\left|V^{\prime \prime}\right| \psi_{1}\right\rangle}{K_{a 1}-K_{a 8}} \psi_{8}+\frac{\left\langle\psi_{9}\left|V^{\prime \prime}\right| \psi_{1}\right\rangle}{K_{a 1}-K_{a 9}} \psi_{9}+\frac{\left\langle\psi_{10}\left|V^{\prime \prime}\right| \psi_{1}\right\rangle}{K_{a 1}-K_{a 10}} \psi_{10} \\
&+K_{a 1}-K_{a 11}
\end{aligned}
$$

Hence, the new wave function is a linear combination of the original eigenfunction and its 
first order correction

$$
\Psi_{1}=\psi_{1}+\psi_{1}^{(1)}=\psi_{1}+\varepsilon_{1} \psi_{1}^{\prime}+\varepsilon_{2} \psi_{1}^{\prime \prime}
$$

(where we replace the indices $n_{a}^{\prime}, l^{\prime}, n^{\prime}, m^{\prime}$ by the index $j$, in the same way we replace the indices $n_{a}, l, n, m$ by the index $i$, according to the table in page 6 )

We wish to calculate the Berry phase produced by the new wave function. For that purpose we first evaluate the formula of the Berry phase for the case of the perturbed four dimensional harmonic oscillator and then we will apply this formula to compute the particular Berry phase produced by the wave function $\psi_{1}$

We take the parameter space to be $\vec{R}=\vec{R}\left(\varepsilon_{1}, \varepsilon_{2}\right)$ where $\varepsilon_{1}, \varepsilon_{2}$ are the coupling parameters of the perturbation. Hence

$$
\begin{aligned}
\left\langle\Psi_{j} \mid \nabla_{\vec{R}} \Psi_{j}\right\rangle & =\left\langle\psi_{j}+\varepsilon_{1} \psi_{j}^{\prime}+\varepsilon_{2} \psi_{j}^{\prime \prime} \mid \psi_{j}^{\prime} \hat{\varepsilon_{1}}+\psi_{j}^{\prime \prime} \hat{\varepsilon_{2}}\right\rangle \\
& =\left\langle\psi_{j} \mid \psi_{j}^{\prime}\right\rangle \hat{\varepsilon_{1}}+\left\langle\psi_{j} \mid \psi_{j}^{\prime \prime}\right\rangle \hat{\varepsilon_{2}} \\
& +\varepsilon_{1}\left\langle\psi_{j}^{\prime} \mid \psi_{j}^{\prime}\right\rangle \hat{\varepsilon_{1}}+\varepsilon_{1}\left\langle\psi_{j}^{\prime} \mid \psi_{j}^{\prime \prime}\right\rangle \hat{\varepsilon_{2}} \\
& +\varepsilon_{2}\left\langle\psi_{j}^{\prime \prime} \mid \psi_{j}^{\prime}\right\rangle \hat{\varepsilon_{1}}+\varepsilon_{2}\left\langle\psi_{j}^{\prime \prime} \mid \psi_{j}^{\prime \prime}\right\rangle \hat{\varepsilon_{2}}
\end{aligned}
$$

where $\hat{\varepsilon_{1}}$ and $\hat{\varepsilon_{2}}$ are unit vectors in the parameter space.

Now, let us define

$$
\psi_{j}^{\prime}=\sum_{i \neq j} a_{i} \psi_{i} \quad \psi_{j}^{\prime \prime}=\sum_{i \neq j} b_{i} \psi_{i}
$$

Hence, from the orthonormality of these eigenfunctions,

$$
\left\langle\psi_{j} \mid \psi_{j}^{\prime}\right\rangle=\left\langle\psi_{j} \mid \sum_{i \neq j} a_{i} \psi_{i}\right\rangle=\sum_{i \neq j} a_{i}\left\langle\psi_{j} \mid \psi_{i}\right\rangle=0
$$

and similarly

$$
\left\langle\psi_{j} \mid \psi_{j}^{\prime \prime}\right\rangle=0
$$

We also have that

$$
\left\langle\psi_{j}^{\prime} \mid \psi_{j}^{\prime}\right\rangle=\left\langle\sum_{i \neq j} a_{i} \psi_{i} \mid \sum_{k \neq j} a_{k} \psi_{k}\right\rangle=\sum_{i \neq j} a_{i}^{*} \sum_{k \neq j} a_{k}\left\langle\psi_{i} \mid \psi_{k}\right\rangle=\sum_{i \neq j} a_{i}^{*} \sum_{k \neq j} a_{k} \delta_{i k}=\sum_{i \neq j}\left|a_{i}\right|^{2}
$$


and similarly

$$
\begin{gathered}
\left\langle\psi_{j}^{\prime \prime} \mid \psi_{j}^{\prime \prime}\right\rangle=\sum_{i \neq j}\left|b_{i}\right|^{2} \\
\left\langle\psi_{j}^{\prime} \mid \psi_{j}^{\prime \prime}\right\rangle=\left\langle\sum_{i \neq j} a_{i} \psi_{i} \mid \sum_{k \neq j} b_{k} \psi_{k}\right\rangle=\sum_{i \neq j} a_{i}^{*} \sum_{k \neq j} b_{k}\left\langle\psi_{i} \mid \psi_{k}\right\rangle=\sum_{i \neq j} a_{i}^{*} \sum_{k \neq j} b_{k} \delta_{i k}=\sum_{i \neq j} a_{i}^{*} b_{i}
\end{gathered}
$$

Substituting these results into Eq.(27), we get

$$
\left\langle\Psi_{j} \mid \nabla_{\vec{R}} \Psi_{j}\right\rangle=\left[\varepsilon_{1} \sum_{i \neq j}\left|a_{i}\right|^{2}+\varepsilon_{2} \sum_{i \neq j} a_{i} b_{i}^{*}\right] \hat{\varepsilon_{1}}+\left[\varepsilon_{1} \sum_{i \neq j} a_{i}^{*} b_{i}+\varepsilon_{2} \sum_{i \neq j}\left|b_{i}\right|^{2}\right] \hat{\varepsilon_{2}}
$$

Now, since $\vec{R}=\vec{R}\left(\varepsilon_{1}, \varepsilon_{2}\right)$ then

$$
d \vec{R}=d \varepsilon_{1} \hat{\varepsilon_{1}}+d \varepsilon_{2} \hat{\varepsilon_{2}}
$$

Hence

$$
\left\langle\Psi_{j} \mid \nabla_{\vec{R}} \Psi_{j}\right\rangle \cdot d \vec{R}=\left[\varepsilon_{1} \sum_{i \neq j}\left|a_{i}\right|^{2}+\varepsilon_{2} \sum_{i \neq j} a_{i} b_{i}^{*}\right] d \varepsilon_{1}+\left[\varepsilon_{1} \sum_{i \neq j} a_{i}^{*} b_{i}+\varepsilon_{2} \sum_{i \neq j}\left|b_{i}\right|^{2}\right] d \varepsilon_{2}
$$

Now, let us assume that the adiabatic change of the parameters $\varepsilon_{1}$ and $\varepsilon_{2}$ follow a circle (with small radius $r$ ) in the parameter space, so we take $\varepsilon_{1}=r \cos \alpha$ and $\varepsilon_{2}=r \sin \alpha$. From Eq.(27) and Eq.(17), we obtain

$$
\begin{aligned}
\gamma_{j}=i r^{2}[ & -\sum_{i \neq j}\left|a_{i}\right|^{2} \int_{0}^{2 \pi} \cos \alpha \sin \alpha d \alpha-\sum_{i \neq j} a_{i} b_{i}^{*} \int_{0}^{2 \pi} \sin ^{2} \alpha d \alpha \\
& \left.+\sum_{i \neq j} a_{i}^{*} b_{i} \int_{0}^{2 \pi} \cos ^{2} \alpha d \alpha+\sum_{i \neq j}\left|b_{i}\right|^{2} \int_{0}^{2 \pi} \sin \alpha \cos \alpha d \alpha\right]
\end{aligned}
$$

Noting that the first and fourth integrals in this expression are equal to zero, and that the second and third integrals are equal to $\pi$, we get

$$
\gamma_{j}=i \pi r^{2} \sum_{i \neq j}\left(a_{i}^{*} b_{i}-a_{i} b_{i}^{*}\right)=-2 \pi r^{2} \sum_{i \neq j} \operatorname{Im}\left(a_{i}^{*} b_{i}\right)=-2 \pi r^{2} \operatorname{Im}\left\{\sum_{i \neq j} a_{i}^{*} b_{i}\right\}
$$

or, in terms of the perturbed wavefunction (by (33)) we find that

$$
\gamma_{j}=-2 \pi r^{2} \operatorname{Im}\left\langle\psi_{j}^{\prime} \mid \psi_{j}^{\prime \prime}\right\rangle
$$




\begin{tabular}{|c|c|c|}
\hline$j$ & $\omega$ & $\gamma_{j} / r^{2}$ \\
\hline 1 & $240.4 \mathrm{MHz}$ & 1.057 \\
\hline 2 & $89.6 \mathrm{MHz}$ & 6.429 \\
\hline 5 & $240.4 \mathrm{MHz}$ & 2.470 \\
\hline 6 & $240.4 \mathrm{MHz}$ & 2.095 \\
\hline 8 & $334.02 \mathrm{MHz}$ & 2.840 \\
\hline 9 & $240.4 \mathrm{MHz}$ & 7.905 \\
\hline 10 & $89.6 \mathrm{MHz}$ & 6.429 \\
\hline 13 & $240.4 \mathrm{mHz}$ & 2.470 \\
\hline 14 & $240.4 \mathrm{MHz}$ & 2.095 \\
\hline 16 & $334.02 \mathrm{MHz}$ & 2.840 \\
\hline
\end{tabular}

TABLE I. Table of angular frequencies and geometric phases

This result can obtained directly from (27) by noting that $d R=-r \sin \alpha d \alpha \hat{\varepsilon_{1}}+r \cos \alpha d \alpha \hat{\varepsilon_{2}}$; therefore only terms in $\varepsilon_{1} d \varepsilon_{2}$ and $\varepsilon_{2} d \varepsilon_{1}$ can contribute to the integral. The result is exactly as given in (39). We have given the orthogonal expansion method as well since it seems instructive to do so.

Hence, the covariant Berry phase, produced by the wave function $\psi_{1}$ (for example) of the perturbed harmonic oscillator, is

$$
\gamma_{1} / r^{2}=-2 \pi \operatorname{Im}\left\langle\psi_{1}^{\prime} \mid \psi_{1}^{\prime \prime}\right\rangle=1.057
$$

Formula (39) contains a scalar product on a given orientation of the RMS, say, $n^{\mu}=$ $(0,0,0,1)$, labeling the defining representation according to Figure 1. A Lorentz transformation of the system induces a transformation of coordinates in $O(2,1)$ of the RMS (the "little group" of the induced representation [2]) and a reorientation along the orbit. The perturbation in Eq. (18) does not contain $n^{\mu}$ explicitly, and therefore the perturbed wave functions depend on $n^{\mu}$ only through the coordinatization of the RMS.

In the scalar product (39), one may make a change of variables to compensate for the induced $O(2,1)$ transformation on the variables of the RMS. The z component, oriented in 
any frame along the $n^{\mu}$ axis, is invariant, and therefore the scalar product defines a set of invariant phases $\gamma_{n}$

We have demonstrated the existence of invariant Berry phases in a relativistically covariant four dimensional harmonic oscillator. As we showed, these phases must be associated with fractional angle perturbation of the azimuthal symmetry of the oscillator. As can be expected, the Berry phase is zero for each eigenstate with $l<n\left(\operatorname{so} P_{l}^{n}(\cos \theta)=0\right.$ and hence, the oscillator's eigenfunction is identically zero) or $m<n\left(\right.$ so $P_{m}^{n}(\tanh \beta)=0$ and hence, the first order correction to the oscillator's eigenfunction is equal to zero), and is nonzero for all the other eigenstates (where $l \geqslant n$ and $m \geqslant n$ ), We can see their specific values in Table 1 .

We have shown, furthermore, that, since the perturbing potential does not depend explicitly on the orientation of the RMS according to $n^{\mu}$, the Berry phases we have computed are Lorentz invariant.

[1] R. Arshansky and L. P. Horwitz. The quantum relativistic two-body bound state. i. the spectrum. J. Math. Phys., 30(1):66-80, 1989.

[2] R. Arshansky and L. P. Horwitz. The quantum relativistic two-body bound state. ii. the induced representation of sl(2,c). J. Math. Phys, 30(2):380-392, 1989.

[3] M. V. Berry. Quantal phase factors accompanying adiabatic changes. Proc. R. Lond., A392:45$57,1984$.

[4] J. L. Cook. Aust. J. Phys, 25:141, 1972.

[5] D. J. Griffiths. Introduction to Quantum Mechanics. Benjamin Cummings, 2004.

[6] L. P. Horwitz and C. Piron. Helv. Phys. Acta., 46:316, 1973.

[7] Note that some of the entries in this table, those for which $n \geqslant l$, vanish identically and therefore do not appear in any calculation. We list them for completeness among the indices.

[8] E.C.G. Stueckelberg. Helv. Phys. Acta, 14:372, 588, 1941.

[9] J. S. Zmuidzinas. J. Math. Phys., 7:764, 1966. 